\title{
Effect of Diffusion and Cross-Diffusion in a Predator-Prey Model with a Transmissible Disease in the Predator Species
}

\author{
Guohong Zhang and Xiaoli Wang \\ School of Mathematics and Statistics, Southwest University, Chongqing 400715, China \\ Correspondence should be addressed to Guohong Zhang; zgh711@swu.cn
}

Received 16 September 2013; Accepted 27 February 2014; Published 3 June 2014

Academic Editor: Francisco Solis

Copyright (C) 2014 G. Zhang and X. Wang. This is an open access article distributed under the Creative Commons Attribution License, which permits unrestricted use, distribution, and reproduction in any medium, provided the original work is properly cited.

We study a Lotka-Volterra type predator-prey model with a transmissible disease in the predator population. We concentrate on the effect of diffusion and cross-diffusion on the emergence of stationary patterns. We first show that both self-diffusion and crossdiffusion can not cause Turing instability from the disease-free equilibria. Then we find that the endemic equilibrium remains linearly stable for the reaction diffusion system without cross-diffusion, while it becomes linearly unstable when cross-diffusion also plays a role in the reaction-diffusion system; hence, the instability is driven solely from the effect of cross-diffusion. Furthermore, we derive some results for the existence and nonexistence of nonconstant stationary solutions when the diffusion rate of a certain species is small or large.

\section{Introduction}

The study of the dynamic relationship between predator and prey has long been one of the most important themes in population dynamics because of its universal existence in nature and many different phenomena have been observed (see [1-13] and references therein). At the same time, since species need to interact with the environment, they are always subject to diseases in the natural world. So it is necessary and interesting to combine demographic as well as epidemic aspects in the standard classical population models. This type of systems is now known as ecoepidemic model.

In fact, the importance of disease influence on the dynamics of plant as well as animal populations has been recognized and several such studies are reviewed in a number of recent publications. However, most of the previous researches on ecoepidemic models assume that the distribution of the predators and prey is homogeneous, which leads to the ODE system (see [14-23] and references therein). As we know, both predators and prey have the natural tendency to diffuse to areas of smaller population concentration. At the same time, some prey species always congregate and form a huge group to protect themselves from the attack of infected predator. So it is important to take into account the inhomogeneous distribution of the predators and prey within a fixed bounded domain $\Omega$ and consider the effect of diffusion and crossdiffusion.

In order to construct the corresponding reactiondiffusion type model, we first propose the following assumptions, which are proper in biological background.

(H1) The disease spreads among the predator species only by contact and the disease incidence follows the simple law of mass action.

(H2) In the absence of predators, the prey population $u_{1}$ grows logistically with the intrinsic growth rate $r>$ 0 and carrying capacity $r / B$, in which $B$ measures intraspecific competition of the prey.

(H3) The sound predator population $u_{2}$ has no other food sources, and $\mu>0$ represents natural mortality. The infected predator population $u_{3}$ cannot recover and their total death rate $d>0$ encompasses natural and disease-related mortality. The conversion factor of a consumed prey into a sound or infected predator is $0<e<1$.

(H4) The sound and infected predators hunt the prey with different searching efficiencies, denoted, respectively, 
by $m$ and $p m$, with $0<p<1$. This is due to the fact that sound predators are more efficient to catch the prey than the infected ones, weakened by the infection.

(H5) Both predators and prey have the natural tendency to diffuse to areas of smaller population concentration and the natural dispersive forces of movements of the prey, sound predators, and infected predators are $d_{1}$, $d_{2}$, and $d_{3}$, respectively.

(H6) The prey species congregate and form a huge group to protect themselves from the attack of infected predator.

With the above assumptions, our model takes the following form, in which all parameters are assumed to be positive:

$$
\begin{aligned}
& \frac{\partial u_{1}}{\partial t}-d_{1} \Delta u_{1}=r u_{1}-B u_{1}^{2}-m u_{1} u_{2}-p m u_{1} u_{3} \\
& \text { in } \Omega \times(0, \infty) \text {, } \\
& \frac{\partial u_{2}}{\partial t}-d_{2} \Delta u_{2}=e m u_{1} u_{2}-a u_{2} u_{3}-\mu u_{2} \\
& \text { in } \Omega \times(0, \infty) \text {, } \\
& \frac{\partial u_{3}}{\partial t}-d_{3} \Delta\left(u_{3}+d_{4} u_{1} u_{3}\right)=a u_{2} u_{3}+e m p u_{1} u_{3}-\delta u_{3} \\
& \text { in } \Omega \times(0, \infty) \text {, } \\
& \partial_{\nu} u_{1}=\partial_{\nu} u_{2}=\partial_{\nu} u_{3}=0 \\
& \text { on } \partial \Omega \times(0, \infty) \text {, } \\
& \left(u_{1}(0, x), u_{2}(0, x), u_{3}(0, x)\right) \geq(0,0,0)
\end{aligned}
$$

on $\Omega$,

where $\Omega$ is a bounded domain in $R^{N}(N \geq 1$ is an integer) with a smooth boundary $\partial \Omega$ and $v$ is the outward unit rector on $\partial \Omega$. The homogeneous Neumann boundary condition indicates that there is zero population flux across the boundary. In the diffusion terms, the constant $d_{i}(i=$ $1,2,3)$, which is usually termed self-diffusion coefficient, represents the natural dispersive force of movement of an individual. The constant $d_{3} d_{4}$ could be referred to as crossdiffusion pressure, which describes a mutual interference between individuals.

In fact, it is easy to see that the infected predator $u_{3}$ diffuses with flux:

$$
\begin{aligned}
\mathbf{J} & =-\nabla\left(d_{3} u_{3}+d_{3} d_{4} u_{1} u_{3}\right) \\
& =-d_{3} d_{4} u_{3} \nabla u_{1}-\left(d_{3}+d_{3} d_{4} u_{1}\right) \nabla u_{3} .
\end{aligned}
$$

As $d_{3} d_{4} u_{3}<0$, the part $-d_{3} d_{4} u_{3} \nabla u_{1}$ of the flux is directed toward the decreasing population density of the prey $u_{1}$, which means that the prey species congregate and form a huge group to protect themselves from the attack of infected predator. We remark that this kind of nonlinear diffusion was first introduced by Shigesada et al. [24] and has been used in different type of population models [25-28]. We also point out that the corresponding ODE system of (1) with delay has been studied by [29], and they mainly investigate the stability and bifurcations related to the two most important equilibria of the ecoepidemic system, namely, the endemic equilibrium and the disease-free one.

Since the first example of stationary patterns in a predator-prey system arising solely from the effect of crossdiffusion is introduced by Pang and Wang [30], recently, more attention has been given to investigate the effect of crossdiffusion in reaction-diffusion systems; see, for example, [3136] and references therein. Here we point out that, to our knowledge, there is little work about ecoepidemic models with diffusion and cross-diffusion was discussed.

In our work here, one of the main purposes is to study the existence of positive stationary solutions of (1) by using degree theory, which are the positive solutions of

$$
\begin{gathered}
-d_{1} \Delta u_{1}=r u_{1}-B u_{1}^{2}-m u_{1} u_{2}-p m u_{1} u_{3} \quad \text { in } \Omega, \\
-d_{2} \Delta u_{2}=e m u_{1} u_{2}-a u_{2} u_{3}-\mu u_{2} \quad \text { in } \Omega, \\
-d_{3} \Delta\left(u_{3}+d_{4} u_{1} u_{3}\right)=a u_{2} u_{3}+e m p u_{1} u_{3}-\delta u_{3}
\end{gathered}
$$

in $\Omega$,

$$
\partial_{\nu} u_{1}=\partial_{\nu} u_{2}=\partial_{\nu} u_{3}=0 \quad \text { on } \partial \Omega \text {. }
$$

Hence we are interested in nonconstant positive solutions of (3), which correspond to coexistence states of prey and predators. For convenience, we denote $\Lambda=(r, B, m, p, e, a, \mu, \delta)$. By a direct computation, we can show that (3) has a semitrivial constant steady state $\mathbf{u}_{0}^{*}=\left(u_{01}^{*}, u_{02}^{*}, u_{03}^{*}\right)=(\mu / \mathrm{em},(\mathrm{erm}-$ $\left.\mu B) / \mathrm{em}^{2}, 0\right)$ if $\mathrm{erm}>\mu B$ and has a positive constant steady state $\mathbf{u}^{*}=\left(u_{1}^{*}, u_{2}^{*}, u_{3}^{*}\right)$, where

$$
\begin{gathered}
u_{1}^{*}=\frac{r a-m(\delta-\mu p)}{a B}, \quad u_{2}^{*}=\frac{\delta-e m p u^{*}}{a}, \\
u_{3}^{*}=\frac{e m u^{*}-\mu}{a},
\end{gathered}
$$

provided that

$$
\begin{gathered}
a r>m(\delta-\mu p), \\
\frac{\delta}{e m p}>\frac{r a-m(\delta-\mu p)}{a B}>\frac{\mu}{e m} .
\end{gathered}
$$

Here we remark that the semitrivial constant steady state $\mathbf{u}_{0}^{*}$ and the positive constant steady state $\mathbf{u}^{*}$ are also called disease-free equilibrium and endemic equilibrium, respectively, in endemic models.

The rest of this paper is organized as follows. In Section 2, we will investigate the stability of disease-free equilibrium $\mathbf{u}_{0}^{*}$ and the endemic equilibrium $\mathbf{u}^{*}$ and show that the cross-diffusion destabilizes a uniform equilibrium which is stable for the kinetic and self-diffusion reaction systems. In Section 3, a priori upper bounds and lower bounds for the nonconstant positive solutions of (3) are given. In Section 4, 
we study nonexistence of nonconstant positive solutions of model (3) when considering only the self-diffusion. Finally, in Section 5, we investigate the existence of the nonconstant positive solutions of (3) by using the Leray-Schauder degree theory, which explains why shrub ecosystem generates patterns.

\section{Stability Analysis of the Constant Solutions $\mathbf{u}_{0}^{*}$ and $\mathbf{u}^{*}$}

In order to study the stability of the constant steady states $\mathbf{u}_{0}^{*}$ and $\mathbf{u}^{*}$ of (1), we first set up the following notation.

Notation 1. Consider the following.

(i) $0=\mu_{0}<\mu_{1}<\mu_{2}<\cdots$ are the eigenvalues of $-\Delta$ in $\Omega$ under homogeneous Neumann boundary condition.

(ii) $S\left(\mu_{i}\right)$ is the set of eigenfunctions corresponding to $\mu_{i}$.

(iii) $\mathbf{X}_{i j}:=\mathbf{c} \varphi_{i j}: \mathbf{c} \in \mathbb{R}^{3}$, where $\varphi_{i j}$ are orthonormal basis of $S\left(\mu_{i}\right)$ for $j=1, \ldots, \operatorname{dim}\left[S\left(\mu_{i}\right)\right]$.

(iv) $\mathbf{X}:=\left\{\left(u_{1}, u_{2}, u_{3}\right) \in\left[C^{1}(\bar{\Omega})\right]^{3}: \partial u_{1} / \partial v=\partial u_{2} / \partial v=\right.$ $\partial u_{3} / \partial \nu=0$ on $\left.\partial \Omega\right\}$, and so $\mathbf{X}=\oplus_{i=1}^{\infty} \oplus_{j=1}^{\operatorname{dim}\left[S\left(\mu_{i}\right)\right]} \mathbf{X}_{i j}$.

Now, we first consider system (1) without cross-diffusion and introduce the following system:

$$
\begin{aligned}
& \frac{\partial u_{1}}{\partial t}-d_{1} \Delta u_{1}=r u_{1}-B u_{1}^{2}-m u_{1} u_{2}-p m u_{1} u_{3} \\
& \text { in } \Omega \times(0, \infty) \text {, } \\
& \frac{\partial u_{2}}{\partial t}-d_{2} \Delta u_{2}=e m u_{1} u_{2}-a u_{2} u_{3}-\mu u_{2} \\
& \text { in } \Omega \times(0, \infty) \text {, } \\
& \frac{\partial u_{3}}{\partial t}-d_{3} \Delta u_{3}=a u_{2} u_{3}+e m p u_{1} u_{3}-\delta u_{3} \\
& \text { in } \Omega \times(0, \infty) \text {, } \\
& \partial_{\nu} u_{1}=\partial_{\nu} u_{2}=\partial_{\nu} u_{3}=0 \\
& \text { on } \partial \Omega \times(0, \infty) \text {, } \\
& \left(u_{1}(0, x), u_{2}(0, x), u_{3}(0, x)\right) \geq(0,0,0) \\
& \text { on } \Omega \text {. }
\end{aligned}
$$

Obviously, system (6) has the same disease-free equilibrium $\mathbf{u}_{0}^{*}$ and endemic equilibrium $\mathbf{u}^{*}$ with system (1). From (6), we can get the following theorem.

Theorem 1. (i) If erm $>\mu B$ and $(\mathrm{erm}+\mu B) / \mathrm{em}^{2}<$ $\delta / a$, the disease-free equilibrium $\mathbf{u}_{0}^{*}$ of system (6) is locally asymptotically stable.

(ii) Assume that (5) holds. The endemic equilibrium $\mathbf{u}^{*}$ of system (6) is locally asymptotically stable.
Proof. (i) For simplicity, throughout this paper, we denote

$$
\begin{aligned}
\mathbf{G}(\mathbf{u}) & =\left(\begin{array}{l}
G_{1}(\mathbf{u}) \\
G_{2}(\mathbf{u}) \\
G_{3}(\mathbf{u})
\end{array}\right) \\
& =\left(\begin{array}{c}
r u_{1}-B u_{1}^{2}-m u_{1} u_{2}-p m u_{1} u_{3} \\
e m u_{1} u_{2}-a u_{2} u_{3}+\mu u_{2} \\
a u_{2} u_{3}+e m p u_{1} u_{3}-\delta u_{3}
\end{array}\right) .
\end{aligned}
$$

By a direct calculation, we obtain

$$
\mathbf{G}_{\mathbf{u}}\left(\mathbf{u}_{0}^{*}\right)=\left(\begin{array}{ccc}
-B u_{1}^{*} & -m u_{01}^{*} & -p m u_{01}^{*} \\
e m u_{02}^{*} & 0 & -a u_{02}^{*} \\
0 & 0 & a u_{02}^{*}-\delta
\end{array}\right)
$$

The linearization of (6) at $\mathbf{u}_{0}^{*}$ can be expressed by

$$
\mathbf{u}_{t}=\left(\mathscr{D} \Delta+\mathbf{G}_{\mathbf{u}}\left(\mathbf{u}_{0}^{*}\right)\right) \mathbf{u},
$$

where

$$
\mathscr{D}=\left(\begin{array}{ccc}
d_{1} & 0 & 0 \\
0 & d_{2} & 0 \\
0 & 0 & d_{3}
\end{array}\right)
$$

According to Notation 1, $\mathbf{X}_{i}$ is invariant under the operator $\mathscr{D} \Delta+\mathbf{G}_{\mathbf{u}}\left(\mathbf{u}_{0}^{*}\right)$, and $\lambda$ is an eigenvalue of this operator on $\mathbf{X}_{i}$ if and only if it is an eigenvalue of the matrix $-\mu_{i} \mathscr{D}+\mathbf{G}_{\mathbf{u}}\left(\mathbf{u}_{0}^{*}\right)$.

A direct calculation shows that the characteristic polynomial of $-\mu_{i} \mathscr{D}+\mathbf{G}_{\mathbf{u}}\left(\mathbf{u}_{0}^{*}\right)$ can be given by

$$
\begin{aligned}
\phi_{0 i}(\lambda)= & \left(\lambda+\delta-a u_{02}^{*}+d_{3} \mu_{i}\right) \\
& \times\left[\lambda^{2}+\left(B u_{01}^{*}+d_{1} \mu_{i}+d_{2} \mu_{i}\right) \lambda+e m^{2} u_{01}^{*} u_{02}^{*}\right] .
\end{aligned}
$$

It follows from (11) that, if $(\mathrm{erm}+\mu B) / \mathrm{em}^{2}<\delta / a$, the corresponding eigenvalues have negative real parts for all $i \geq$ 1 , so we know that $u_{0}^{*}$ is locally asymptotically stable.

(ii) Since $\mathbf{G}\left(\mathbf{u}^{*}\right)=0$, it follows from (7) that

$$
\mathbf{G}_{\mathbf{u}}\left(\mathbf{u}^{*}\right)=\left(\begin{array}{ccc}
-B u_{1}^{*} & -m u_{1}^{*} & -p m u_{1}^{*} \\
e m u_{2}^{*} & 0 & -a u_{2}^{*} \\
e m p u_{3}^{*} & a u_{3}^{*} & 0
\end{array}\right)
$$

The linearization of (6) at $\mathbf{u}^{*}$ can be expressed by

$$
\mathbf{u}_{t}=\left(\mathscr{D} \Delta+\mathbf{G}_{\mathbf{u}}\left(\mathbf{u}^{*}\right)\right) \mathbf{u},
$$

where the matrix $\mathscr{D}$ is defined in (10). Direct calculation shows that the characteristic polynomial of $-\mu_{i} \mathscr{D}+\mathbf{G}_{\mathbf{u}}\left(\mathbf{u}^{*}\right)$ is given by

$$
\phi_{i}(\lambda)=\lambda^{3}+c_{1} \lambda^{2}+c_{2} \lambda+c_{3}
$$


where

$$
\begin{gathered}
c_{1}=c_{1}\left(\mu_{i}\right)=\left(d_{1}+d_{2}+d_{3}\right) \mu_{i}+B u_{1}^{*}, \\
c_{2}=c_{2}\left(\mu_{i}\right)=\left(d_{1} d_{2}+d_{1} d_{3}+d_{2} d_{3}\right) \mu_{i}^{2}+\left(d_{2}+d_{3}\right) B u_{1}^{*} \mu_{i} \\
+\left(e m^{2} u_{1}^{*} u_{2}^{*}+e m^{2} p^{2} u_{1}^{*} u_{3}^{*}+a^{2} u_{2}^{*} u_{3}^{*}\right), \\
c_{3}=c_{3}\left(\mu_{i}\right)=d_{1} d_{2} d_{3} \mu_{i}^{3}+d_{2} d_{3} B u_{1}^{*} \mu_{i}^{2} \\
+\left(d_{1} a^{2} u_{2}^{*} u_{3}^{*}+d_{2} e m^{2} p^{2} u_{1}^{*} u_{3}^{*}+d_{3} e^{2} u_{1}^{*} u_{2}^{*}\right) \mu_{i} \\
+B a^{2} u_{1}^{*} u_{2}^{*} u_{3}^{*} .
\end{gathered}
$$

It is easy to see that $c_{1}, c_{2}$, and $c_{3}$ are positive.

Notice that

$$
\begin{aligned}
c_{1} c_{2}-c_{3}= & \left(d_{2}+d_{3}\right)\left(d_{1}^{2}+d_{1} d_{2}+d_{1} d_{3}+d_{2} d_{3}\right) \mu_{i}^{3} \\
+ & B u_{1}^{*}\left(d_{2}+d_{3}\right)\left(2 d_{1}+d_{2}+d_{3}\right) \mu_{i}^{2} \\
+ & {\left[\left(B u_{1}^{*}\right)^{2}\left(d_{2}+d_{3}\right)\right.} \\
& +d_{1}\left(e m^{2} u_{1}^{*} u_{2}^{*}+e m^{2} p^{2} u_{1}^{*} u_{3}^{*}\right) \\
& +d_{2}\left(e m^{2} u_{1}^{*} u_{2}^{*}+a^{2} u_{2}^{*} u_{3}^{*}\right) \\
& \left.+d_{3}\left(e m^{2} p^{2} u_{1}^{*} u_{3}^{*}+a^{2} u_{2}^{*} u_{3}^{*}\right)\right] \mu_{i} \\
& +B u_{1}^{*}\left(e m^{2} u_{1}^{*} u_{2}^{*}+e m^{2} p^{2} u_{1}^{*} u_{3}^{*}\right)>0 .
\end{aligned}
$$

Then by the Routh-Hurwitz criterion, we know that, for each $i \geq 1$, all the three roots $\lambda_{i, 1}, \lambda_{i, 2}$, and $\lambda_{i, 3}$ of characteristic equation $\phi_{i}(\lambda)=0$ have negative real parts. Now we can prove that there exists a positive constant $\delta$ such that

$$
\operatorname{Re}\left\{\lambda_{i 1}\right\}, \quad \operatorname{Re}\left\{\lambda_{i 1}\right\}, \quad \operatorname{Re}\left\{\lambda_{i 1}\right\} \leq-\delta, \quad i \geq 1 .
$$

In fact, let $\lambda=\mu_{i} \xi$; then we have

$$
\phi_{i}(\lambda)=\mu_{i}^{3} \xi^{3}+c_{1} \mu_{i}^{2} \xi^{2}+c_{2} \mu_{i} \xi+c_{3} \triangleq \widetilde{\phi}_{i}(\xi) .
$$

Note that $\mu_{i} \mapsto \infty$ as $i \mapsto \infty$. It follows that

$$
\begin{aligned}
\lim _{i \mapsto \infty} \frac{\widetilde{\phi}_{i}(\xi)}{\mu_{i}^{3}}= & \xi^{3}+\left(d_{1}+d_{2}+d_{3}\right) \xi^{2} \\
& +\left(d_{1} d_{2}+d_{1} d_{3}+d_{2} d_{3}\right) \xi+d_{1} d_{2} d_{3} \triangleq \widetilde{\phi}(\xi) .
\end{aligned}
$$

Using the Routh-Hurwitz criterion again, we can see that all the three roots $\xi_{1}, \xi_{2}$, and $\xi_{3}$ of equation $\widetilde{\phi}(\xi)=0$ have negative real parts. Thus, there exists a positive constant such that

$$
\operatorname{Re}\left\{\xi_{1}\right\}, \quad \operatorname{Re}\left\{\xi_{2}\right\}, \quad \operatorname{Re}\left\{\xi_{3}\right\} \leq-\bar{\delta} .
$$

By continuity, we know that there exists $i_{0} \in N$ such that the three roots $\xi_{i 1}, \xi_{i 2}$, and $\xi_{i 3}$ of $\widetilde{\phi}_{i}(\xi)=0$ satisfy

$$
\operatorname{Re}\left\{\xi_{i 1}\right\}, \quad \operatorname{Re}\left\{\xi_{i 2}\right\}, \quad \operatorname{Re}\left\{\xi_{i 3}\right\} \leq \frac{-\bar{\delta}}{2}, \quad i \geq i_{0},
$$

which implies that

$$
\operatorname{Re}\left\{\lambda_{i 1}\right\}, \quad \operatorname{Re}\left\{\lambda_{i 2}\right\}, \quad \operatorname{Re}\left\{\lambda_{i 3}\right\} \leq \frac{-\mu_{i} \bar{\delta}}{2} \leq \frac{-\bar{\delta}}{2}, \quad i \geq i_{0} .
$$

Let

$$
-\widetilde{\delta}=\max _{1 \leq i \leq i_{0}}\left\{\operatorname{Re}\left\{\lambda_{i 1}\right\}, \operatorname{Re}\left\{\lambda_{i 2}\right\}, \operatorname{Re}\left\{\lambda_{i 3}\right\}\right\} ;
$$

then $\widetilde{\delta}>0$, and (17) holds for $\delta=\min \{\tilde{\delta}, \bar{\delta} / 2\}$. Thus the proof is completed by Theorem 5.1.1 of Henry [37].

Remark 2. From Theorem 1, we can see that if only the free diffusion is introduced to the corresponding ODE system of (1), the uniform positive stationary solution is also locally stable, which means that only self-diffusion cannot induce Turing instability.

We now consider the effect of the cross-diffusion and introduce the following theorem, which give the necessary conditions for the existence of nonconstant positive solution of system (3).

Theorem 3. Consider the following.

(i) If erm $>\mu B$ and $(\mathrm{erm}+\mu B) / \mathrm{em}^{2}<\delta / a$, the disease-free equilibrium $\mathbf{u}_{0}^{*}$ of system (1) is locally asymptotically stable.

(ii) Assume that (5) holds and $d_{4}>0$ in (1). Suppose that $b_{2}<0$ and $b_{2}^{2}-4 b_{1} b_{3}>0$, where $b_{i}$ is given in (31). If $\mu_{2}^{*} \in\left(\mu_{i}, \mu_{i+1}\right)$ and $\mu_{3}^{*} \in\left(\mu_{j}, \mu_{j+1}\right)$ for some $j>i>$ 0 , where $\mu_{2}^{*}$ and $\mu_{3}^{*}$ are defined in (34), there exists a positive constant $d_{3}^{*}$ such that the uniform stationary solution $\mathbf{u}^{*}$ of (1) is unstable when $d_{3} \geq d_{3}^{*}$.

Proof. (i) For simplicity, we denote that $\Phi(\mathbf{u})=\left(d_{1} u_{1}, d_{2} u_{2}\right.$, $\left.d_{3}\left(u_{3}+d_{4} u_{4}\right)\right)^{\mathrm{T}}$. Then the linearized system of system (1) at $\mathbf{u}_{0}^{*}$ is

$$
\mathbf{u}_{t}=\left(\Phi_{\mathbf{u}}\left(\mathbf{u}_{0}^{*}\right) \Delta+\mathbf{G}_{\mathbf{u}}\left(\mathbf{u}_{0}^{*}\right)\right) \mathbf{u},
$$

where

$$
\Phi_{\mathbf{u}}\left(\mathbf{u}_{0}^{*}\right)=\left(\begin{array}{ccc}
d_{1} & 0 & 0 \\
0 & d_{2} & 0 \\
0 & 0 & d_{3}+d_{3} d_{4} u_{01}^{*}
\end{array}\right) .
$$

By some calculations, the characteristic polynomial of $-\mu_{i} \Phi_{\mathbf{u}}\left(\mathbf{u}_{0}^{*}\right)+\mathbf{G}_{\mathbf{u}}\left(\mathbf{u}_{0}^{*}\right)$ can be given by

$$
\begin{aligned}
\psi_{0 i}(\lambda)= & \left(\lambda+\delta-a u_{02}^{*}+d_{3} \mu_{i}+d_{3} d_{4} u_{01}^{*} \mu_{i}\right) \\
& \times\left(\lambda^{2}+\left(B u_{01}^{*}+d_{1} \mu_{i}+d_{2} \mu_{i}\right) \lambda+e m^{2} u_{01}^{*} u_{02}^{*}\right) .
\end{aligned}
$$

It is easy to see that, if $(\mathrm{erm}+\mu B) / \mathrm{em}^{2}<\delta / a$, all the corresponding eigenvalues of $\phi_{0 i}(\lambda)=0$ have negative real parts for all $i \geq 1$, which implies that $u_{0}^{*}$ is locally asymptotically stable. 
(ii) The linearized system of system (1) at $\mathbf{u}^{*}$ is

$$
\mathbf{u}_{t}=\left(\Phi_{\mathbf{u}}\left(\mathbf{u}^{*}\right) \Delta+\mathbf{G}_{\mathbf{u}}\left(\mathbf{u}^{*}\right)\right) \mathbf{u},
$$

where

$$
\Phi_{\mathbf{u}}\left(\mathbf{u}^{*}\right)=\left(\begin{array}{ccc}
d_{1} & 0 & 0 \\
0 & d_{2} & 0 \\
d_{3} d_{4} u_{3}^{*} & 0 & d_{3}+d_{3} d_{4} u_{1}^{*}
\end{array}\right)
$$

By some calculations, the characteristic polynomial of $-\mu_{i} \Phi_{\mathbf{u}}\left(\mathbf{u}^{*}\right)+\mathbf{G}_{\mathbf{u}}\left(\mathbf{u}^{*}\right)$ can be given by

$$
\Psi_{i}(\lambda)=\lambda^{3}+\bar{c}_{1} \lambda^{2}+\bar{c}_{2} \lambda+\bar{c}_{3},
$$

where

$$
\begin{gathered}
\bar{c}_{1}=\left(d_{1}+d_{2}+d_{3}+d_{3} d_{4} u_{1}^{*}\right) \mu_{i}+B u_{1}^{*}, \\
\bar{c}_{2}=\left[d_{1} d_{2}+d_{1} d_{3}+d_{2} d_{3}+\left(d_{1} d_{3} d_{4}+d_{2} d_{3} d_{4}\right) u_{1}^{*}\right] \mu_{i}^{2} \\
+\left[B\left(d_{2}+d_{3}+d_{3} d_{4}\right)\left(u_{1}^{*}\right)^{2}-d_{3} d_{4} p m u_{1}^{*} u_{2}^{*}\right] \mu_{i} \\
+e m^{2} u_{1}^{*} u_{2}^{*}+e m^{2} p^{2} u_{1}^{*} u_{3}^{*}+a^{2} u_{2}^{*} u_{3}^{*}, \\
\bar{c}_{3}=d_{1} d_{2} d_{3}\left(1+d_{4} u_{1}^{*}\right) \mu_{i}^{3} \\
+\left[B u_{1}^{*}\left(d_{2} d_{3}+d_{2} d_{3} d_{4} u_{1}^{*}\right)-d_{2} d_{3} d_{4} p m u_{1}^{*} u_{3}^{*}\right] \mu_{i}^{2} \\
+\left[\left(d_{1} a^{2} u_{2}^{*} u_{3}^{*}\right)+d_{2} e m^{2} p^{2} u_{1}^{*} u_{3}^{*}\right. \\
\left.+\left(d_{3}+d_{3} d_{4} u_{1}^{*}\right) e m^{2} u_{1}^{*} u_{2}^{*}+d_{3} d_{4} a m u_{1}^{*} u_{2}^{*} u_{3}^{*}\right] \mu_{i} \\
+B a^{2} u_{1}^{*} u_{2}^{*} u_{3}^{*} \\
\triangleq Q_{3} \mu_{i}^{3}+Q_{2} \mu_{i}^{2}+Q_{1} \mu_{i}+Q_{0} .
\end{gathered}
$$

Let $\lambda_{i, 1}, \lambda_{i, 2}$, and $\lambda_{i, 3}$ be the three roots of $\Psi_{i}(\lambda)=0$; then $\lambda_{i, 1} \lambda_{i, 2} \lambda_{i, 3}=-\bar{c}_{3}$. In order to have at least one $\operatorname{Re} \lambda_{i, j}>0(j=$ $1,2,3)$, it is sufficient to prove that $\bar{c}_{3}<0$.

In the following we will find out the conditions such that $\bar{c}_{3}<0$. Let $\widetilde{Q}(\mu)=Q_{3} \mu^{3}+Q_{2} \mu^{2}+Q_{1} \mu+Q_{0}$ and let $\tilde{\mu}_{1}, \widetilde{\mu}_{2}$, and $\widetilde{\mu}_{3}$ be the three roots of $\widetilde{Q}(\mu)=0$ with $\operatorname{Re}\left(\widetilde{\mu}_{1}\right) \leq \operatorname{Re}\left(\tilde{\mu}_{2}\right) \leq$ $\operatorname{Re}\left(\widetilde{\mu}_{3}\right)$. Notice that $Q_{0}>0$ and $Q_{3}>0$. Then $\widetilde{\mu}_{1} \widetilde{\mu}_{2} \widetilde{\mu}_{3}=$ $-Q_{0} / Q_{3}<0$. Thus, one of the three roots $\tilde{\mu}_{1}, \tilde{\mu}_{2}$, and $\tilde{\mu}_{3}$ is real and negative, and the product of the other two is positive.

Consider the following limits:

$$
\lim _{d_{3} \mapsto \infty} \frac{Q_{0}}{d_{3}}=0,
$$

$$
\begin{gathered}
\lim _{d_{3} \mapsto \infty} \frac{Q_{1}}{d_{3}}=\left(1+d_{4} u_{1}^{*}\right) e m^{2} u_{1}^{*} u_{2}^{*}+d_{4} a m u_{1}^{*} u_{2}^{*} u_{3}^{*} \triangleq b_{1}, \\
\lim _{d_{3} \mapsto \infty} \frac{Q_{2}}{d_{3}}=B u_{1}^{*}\left(d_{2}+d_{2} d_{4} u_{1}^{*}\right)-d_{2} d_{4} p m u_{1}^{*} u_{3}^{*} \triangleq b_{2}, \\
\lim _{d_{3} \mapsto \infty} \frac{Q_{3}}{d_{3}}=d_{1} d_{2}\left(1+d_{4} u_{1}^{*}\right) \triangleq b_{3} .
\end{gathered}
$$

Note that

$$
\lim _{d_{3} \mapsto \infty} \frac{\widetilde{Q}(\mu)}{d_{3}}=b_{3} \mu^{3}+b_{2} \mu^{2}+b_{1} \mu=\mu\left(b_{3} \mu^{2}+b_{2} \mu+b_{1}\right) \text {. }
$$

It follows that equation $b_{3} \mu^{2}+b_{2} \mu+b_{1}=0$ has two strictly positive solutions when the following conditions hold:

$$
b_{2}<0, \quad b_{2}^{2}-4 b_{1} b_{3}>0 .
$$

By a continuity argument, we know that, when $d_{3}$ is large enough, $\widetilde{\mu}_{1}$ is real and negative, and $\widetilde{\mu}_{2}$ and $\widetilde{\mu}_{3}$ are real and positive as $\tilde{\mu}_{2} \widetilde{\mu}_{3}>0$. Furthermore, we have

$$
\begin{gathered}
\lim _{d_{3} \mapsto \infty} \tilde{\mu}_{1}=0, \\
\lim _{d_{3} \mapsto \infty} \tilde{\mu}_{2}=\frac{-b_{2}-\sqrt{b_{2}^{2}-4 b_{1} b_{3}}}{2 b_{3}} \triangleq \mu_{2}^{*}>0, \\
\lim _{d_{3} \mapsto \infty} \tilde{\mu}_{3}=\frac{-b_{2}+\sqrt{b_{2}^{2}-4 b_{1} b_{3}}}{2 b_{3}} \triangleq \mu_{3}^{*}>0 .
\end{gathered}
$$

So there exists a positive number $d_{3}^{*}$ such that, when $d_{3}>d_{3}^{*}$, the following hold:

$$
\begin{gathered}
-\infty<\tilde{\mu}_{1}<0<\widetilde{\mu}_{2}<\tilde{\mu}_{3} ; \\
\widetilde{Q}(\mu)<0 \quad \text { if } \mu \in\left(-\infty, \widetilde{\mu}_{1}\right) \cup\left(\widetilde{\mu}_{2}, \widetilde{\mu}_{3}\right) ; \\
\widetilde{Q}(\mu)>0 \quad \text { if } \mu \in\left(\tilde{\mu}_{1}, \widetilde{\mu}_{2}\right) \cup\left(\widetilde{\mu}_{3}, \infty\right) .
\end{gathered}
$$

Since $\mu_{2}^{*} \in\left(\mu_{i}, \mu_{i+1}\right)$ and $\mu_{3}^{*} \in\left(\mu_{j}, \mu_{j+1}\right)$ for some $j>i>0$, we have $\widetilde{Q}\left(\mu_{k}\right)<0$ when $i+1<k<j+1$. Thus we know that $\bar{c}_{3}<0$, and the proof is completed.

\section{A Priori Estimates to the Positive Solution of $(3)$}

In this section, we will give a priori estimates to the positive solution of (3). Let us first introduce two lemmas and we remark that the first lemma is due to Lou and $\mathrm{Ni}$ [38].

Lemma 4 (maximum principle). Suppose that $g \in C(\bar{\Omega} \times \mathbb{R})$.

(i) Assume that $w \in C^{2}(\Omega) \cap C^{1}(\bar{\Omega})$ and satisfies

$$
\begin{gathered}
\Delta w(x)+g(x, w(x)) \geq 0 \quad \text { in } \Omega, \\
\partial_{\gamma} w \leq 0 \quad \text { on } \partial \Omega .
\end{gathered}
$$

$$
\text { If } w\left(x_{0}\right)=\max _{\bar{\Omega}} w(x) \text {, then } g\left(x_{0}, w\left(x_{0}\right)\right) \geq 0 \text {. }
$$

(ii) Assume that $w \in C^{2}(\Omega) \cap C^{1}(\bar{\Omega})$ and satisfies

$$
\begin{gathered}
\Delta w(x)+g(x, w(x)) \leq 0 \quad \text { in } \Omega, \\
\partial_{\nu} w \geq 0 \quad \text { on } \partial \Omega .
\end{gathered}
$$

If $w\left(x_{0}\right)=\min _{\bar{\Omega}} w(x)$, then $g\left(x_{0}, w\left(x_{0}\right)\right) \leq 0$. 

[39].

Next, we state the second lemma which is due to Lin et al.

Lemma 5 (Harnack inequality). Assume that $c(x) \in C(\bar{\Omega})$. Let $w \in C^{2}(\Omega) \cap C^{1}(\bar{\Omega})$ and satisfy

$$
\begin{gathered}
\Delta w(x)+g(x, w(x))=0 \quad \text { in } \Omega, \\
\partial_{\gamma} w=0 \quad \text { on } \partial \Omega .
\end{gathered}
$$

Then there exists a positive constant $C$, depending only on $\|c(x)\|_{C(\bar{\Omega})}$ such that

$$
\max _{\bar{\Omega}} w(x) \leq C \min _{\bar{\Omega}} w(x) .
$$

Our results are the following theorems.

Theorem 6 (upper bounds). Any positive solution $\mathbf{u}(x)=$ $\left(u_{1}(x), u_{2}(x), u_{3}(x)\right)^{T}$ of (3) satisfies

$$
\begin{gathered}
\max _{\bar{\Omega}} u_{1}(x) \leq \bar{M}_{1}, \quad \max _{\bar{\Omega}} u_{2}(x) \leq \bar{M}_{2}, \\
\max _{\bar{\Omega}} u_{3}(x) \leq \bar{M}_{3},
\end{gathered}
$$

where

$$
\begin{gathered}
\bar{M}_{1}=\frac{r}{B}, \quad \bar{M}_{2}=\frac{e r d_{1}}{B d_{2}}+\frac{e r^{2}}{\mu B} \\
\bar{M}_{3}=\frac{e r}{d_{3} B}\left[d_{1}+e+\frac{d_{2} r}{4 \mu}+\frac{d_{3} r}{4 \delta}\left(1+\frac{d_{4} r}{B}\right)\right] .
\end{gathered}
$$

Proof. Let $x_{0} \in \bar{\Omega}$ such that $u_{1}\left(x_{0}\right)=\max _{\bar{\Omega}} u_{1}(x)$. Then by Lemma 4 , it is clear that

$$
\begin{gathered}
r u_{1}\left(x_{0}\right)-B u_{1}^{2}\left(x_{0}\right)-m u_{1}\left(x_{0}\right) u_{2}\left(x_{0}\right) \\
-p m u_{1}\left(x_{0}\right) u_{3}\left(x_{0}\right) \geq 0
\end{gathered}
$$

and so

$$
u_{1}\left(x_{0}\right)=\max _{\bar{\Omega}} u_{1}(x) \leq \frac{r}{B} \triangleq \bar{M}_{1} .
$$

Define $y(x)=e d_{1} u_{1}(x)+d_{2} u_{2}(x)$; then $y(x)$ satisfies

$$
\begin{gathered}
-\Delta y=e r u_{1}-e B u_{1}^{2}-e p m u_{1} u_{3}-a u_{2} u_{3}-\mu u_{2} \\
\text { in } \Omega, \\
\partial_{v} y=0 \quad \text { on } \partial \Omega .
\end{gathered}
$$

Let $x_{1} \in \bar{\Omega}$ such that $y\left(x_{1}\right)=\max _{\bar{\Omega}} y(x)$. Then, by Lemma 4 , we can get

$$
\begin{gathered}
e r u_{1}\left(x_{1}\right)-e B u_{1}^{2}\left(x_{1}\right)-e p m u_{1}\left(x_{1}\right) u_{3}\left(x_{1}\right) \\
-a u_{2}\left(x_{1}\right) u_{3}\left(x_{1}\right)-\mu u_{2}\left(x_{1}\right) \geq 0
\end{gathered}
$$

which implies

$$
u_{2}\left(x_{1}\right) \leq \frac{1}{\mu}\left(e r u_{1}\left(x_{1}\right)-e B u_{1}^{2}\left(x_{1}\right)\right) \leq \frac{e r^{2}}{4 \mu B} .
$$

So, by the definition of $y(x)$, we have

$$
\begin{aligned}
\max _{\bar{\Omega}} u_{2}(x) & \leq \frac{1}{d_{2}} \max _{\bar{\Omega}} y(x)=\frac{1}{d_{2}} y\left(x_{1}\right) \\
& =\frac{e d_{1}}{d_{2}} u_{1}\left(x_{1}\right)+u_{2}\left(x_{1}\right) \leq \frac{e r d_{1}}{B d_{2}}+\frac{e r^{2}}{4 \mu B} \triangleq \bar{M}_{2} .
\end{aligned}
$$

Let $\omega(x)=u_{3}(x)+d_{4} u_{1}(x) u_{3}(x)$; then $u_{3}(x)=\omega(x) /(1+$ $\left.d_{4} u_{1}(x)\right)$. Define $z(x)=e d_{1} u_{1}(x)+d_{2} u_{2}(x)+d_{3} \omega(x)$; then $z(x)$ satisfies

$$
-\Delta z=e r u_{1}(x)-e B u_{1}^{2}(x)-\mu u_{2}(x)-\frac{\delta \omega(x)}{1+d_{4} u_{1}(x)}
$$

in $\Omega$,

$$
\partial_{\gamma} z=0 \quad \text { on } \partial \Omega
$$

Let $x_{2} \in \bar{\Omega}$ such that $z\left(x_{2}\right)=\max _{\bar{\Omega}} z(x)$. Then, by using Lemma 4 again, we can obtain

$$
e r u_{1}\left(x_{2}\right)-e B u_{1}^{2}\left(x_{2}\right)-\mu u_{2}\left(x_{2}\right)-\frac{\delta \omega\left(x_{2}\right)}{1+d_{4} u_{1}\left(x_{2}\right)} \geq 0
$$

which implies

$$
\begin{aligned}
\omega\left(x_{2}\right) & \leq \frac{1}{\delta}\left(1+d_{4} u_{1}\left(x_{2}\right)\right)\left(e r u_{1}\left(x_{2}\right)-e B u_{1}^{2}\left(x_{2}\right)\right) \\
& \leq \frac{e r^{2}}{4 \delta B}\left(1+\frac{d_{4} r}{B}\right) .
\end{aligned}
$$

It follows that

$$
\begin{aligned}
\max _{\bar{\Omega}} u_{3}(x) & \leq \max _{\bar{\Omega}} \omega(x) \leq \frac{1}{d_{3}} \max _{\bar{\Omega}} z(x)=\frac{1}{d_{3}} z\left(x_{2}\right) \\
& =\frac{1}{d_{3}}\left(e d_{1} u_{1}\left(x_{2}\right)+d_{2} u_{2}\left(x_{2}\right)+d_{3} \omega\left(x_{2}\right)\right) \\
& \leq \frac{e r}{d_{3} B}\left[d_{1}+e+\frac{d_{2} r}{4 \mu}+\frac{d_{3} r}{4 \delta}\left(1+\frac{d_{4} r}{B}\right)\right] \triangleq \bar{M}_{3} .
\end{aligned}
$$

Then we obtain the three upper bounds in (40).

Theorem 7. There exist three positive constants $C_{1}$ (depending on $r / d_{1}, \Omega$ ), $C_{2}$ (depending on emr $/ d_{2} B, \Omega$ ), and $C_{3}$ (depending on $\left.\Lambda, d_{i}, \Omega\right)$ such that any positive solution $\mathbf{u}(x)=$ $\left(u_{1}(x), u_{2}(x), u_{3}(x)\right)$ of (3) satisfies

$$
\begin{gathered}
\frac{\max _{\bar{\Omega}} u_{1}(x)}{\min _{\bar{\Omega}} u_{1}(x)} \leq C_{1}, \\
\frac{\max _{\bar{\Omega}} u_{2}(x)}{\min _{\bar{\Omega}} u_{2}(x)} \leq C_{2}, \\
\frac{\max _{\bar{\Omega}} u_{3}(x)}{\min _{\bar{\Omega}} u_{3}(x)} \leq C_{3}, \quad(i=1,2,3) .
\end{gathered}
$$


Proof. It is easy to see that $u_{i}(x)(i=1,2)$ satisfies

$$
\begin{gathered}
\Delta u_{i}(x)+\frac{c_{i}(x)}{d_{i}} u_{i}(x)=0 \quad \text { in } \Omega, \\
\partial_{\nu} u_{i}(x)=0 \quad \text { on } \partial \Omega,
\end{gathered}
$$

where $c_{1}(x)=r-B u_{1}-m u_{1} u_{2}-p m u_{1} u_{3}$ and $c_{2}(x)=e m u_{1}-$ $a u_{3}-\mu$. By (40), we know that

$$
\left\|\frac{c_{1}(x)}{d_{1}}\right\|_{C(\bar{\Omega})} \leq \frac{r}{d_{1}}, \quad\left\|\frac{c_{2}(x)}{d_{2}}\right\|_{C(\bar{\Omega})} \leq \frac{e m r}{d_{2} B} .
$$

So by Lemma 5, we know that the first two inequalities of (52) hold. Define $\varphi(x)=d_{3} u_{3}(x)+d_{3} d_{4} u_{1}(x) u_{3}(x)$; we have

$$
\begin{gathered}
\Delta \varphi(x)+c_{3}(x) \varphi(x)=0 \quad \text { in } \Omega, \\
\partial_{\nu} \varphi(x)=0 \quad \text { on } \partial \Omega,
\end{gathered}
$$

where $c_{3}(x)=\left(e m p u_{1}+a u_{2}-\delta\right) / d_{3}\left(1+d_{4} u_{1}\right)$. By (40), we know that

$$
\left\|c_{3}(x)\right\|_{C(\bar{\Omega})} \leq \frac{e m p r}{d_{3} B}+\frac{e r d_{1}}{B d_{2} d_{3}}+\frac{e r^{2}}{4 \mu B d_{3}} .
$$

Then Lemma 5 yields $\max _{\bar{\Omega}} \varphi(x) / \min _{\bar{\Omega}} \varphi(x) \leq C_{3}^{*}$ for some positive constant $C_{3}^{*}\left(\Lambda, d_{1}, d_{2}, d_{3}, \Omega\right)$, and

$$
\begin{aligned}
\frac{\max _{\bar{\Omega}} u_{3}}{\min _{\bar{\Omega}} u_{3}} & \leq \frac{\max _{\bar{\Omega}} \varphi \max _{\bar{\Omega}}\left(1+d_{4} u_{1}\right)}{\min _{\bar{\Omega}} \varphi \min _{\bar{\Omega}}\left(1+d_{4} u_{1}\right)} \\
& \leq C_{3}^{*} \frac{\max _{\bar{\Omega}} u_{1}}{\min _{\bar{\Omega}} u_{1}} \leq C_{3}^{*} C_{1} \triangleq C_{3} .
\end{aligned}
$$

The proof is completed.

Theorem 8 (lower bounds). Let $\Lambda, D_{1}, D_{2}, D_{3}$, and $D_{4}$ be fixed positive constants. Assume that

$$
\min \left\{\frac{e m p}{B}\left(r-m \bar{M}_{2}\right), \frac{e m p}{B}\left(r-p m \bar{M}_{3}\right)\right\}>\delta
$$

where $\bar{M}_{2}$ and $\bar{M}_{2}$ are given in (47) and (51). Then there exists a positive constant $\underline{C}=\underline{C}\left(\Lambda, D_{1}, D_{2}, D_{3}, D_{4}\right)$ such that, when $\left(d_{1}, d_{2}, d_{3}\right) \in\left[D_{1}, \infty\right) \times\left[D_{2}, \infty\right) \times\left[D_{3}, \infty\right)$ and $d_{4} \in$ $\left[0, D_{4}\right]$, any positive solution $\mathbf{u}(x)=\left(u_{1}(x), u_{2}(x), u_{3}(x)\right)$ of (3) satisfies

$$
\min _{\bar{\Omega}} u_{i}(x) \geq \underline{C}, \quad i=1,2,3 .
$$

Proof. Suppose that (59) fails. Then there exist sequences $\left\{d_{1, i}, d_{2, i}, d_{3, i}, d_{4, i}\right\}_{i=1}^{\infty}$ with $\left(d_{1, i}, d_{2, i}, d_{3, i}\right) \in\left[D_{1}, \infty\right) \times$ $\left[D_{2}, \infty\right) \times\left[D_{3}, \infty\right)$ and $d_{4, i} \in\left[0, D_{4}\right]$ such that the corresponding positive solutions $\left(u_{1, i}, u_{2, i}, u_{3, i}\right)$ of (3) satisfy

$$
\max _{\bar{\Omega}} u_{1 i} \longmapsto 0, \quad \text { or } \quad \max _{\bar{\Omega}} u_{2 i} \longmapsto 0, \quad \text { or } \quad \max _{\bar{\Omega}} u_{3 i} \longmapsto 0 \text {. }
$$

By a direct application of the maximum principle to the first equation of (3), we can obtain $u_{1 i} \leq r / B$. Integrating by parts, we obtain that

$$
\begin{gathered}
\int_{\Omega}\left(r u_{1 i}-B u_{1 i}^{2}-m u_{1 i} u_{2 i}-p m u_{1 i} u_{3 i}\right) d x=0, \\
\int_{\Omega}\left(e m u_{1} u_{2 i}-a u_{2 i} u_{3 i}-\mu u_{2 i}\right) d x=0, \\
\int_{\Omega}\left(a u_{2 i} u_{3 i}+e m p u_{1 i} u_{3 i}-\delta u_{3 i}\right) d x=0,
\end{gathered}
$$

for $i=1,2, \ldots$. By the standard regularity theorem for the elliptic equations, we know that there exists a subsequence of $\left\{u_{1, i}, u_{2, i}, u_{3, i}\right\}_{i=1}^{\infty}$, which we will still denote by $\left\{u_{1, i}, u_{2, i}, u_{3, i}\right\}_{i=1}^{\infty}$, and three nonnegative functions $u_{1}, u_{2}, u_{3} \in$ $C^{2}(\bar{\Omega})$ such that

$$
\begin{gathered}
\left(u_{1, i}, u_{2, i}, u_{3, i}\right) \longmapsto\left(u_{1}, u_{2}, u_{3}\right) \\
\quad \text { in }\left[C^{2}(\bar{\Omega})\right]^{3} \text { as } i \longmapsto \infty .
\end{gathered}
$$

By (60), we know that

$$
u_{1} \equiv 0, \quad \text { or } \quad u_{2} \equiv 0, \quad \text { or } \quad u_{3} \equiv 0 .
$$

Furthermore, we assume that $\left(d_{1, i}, d_{2, i}, d_{3, i}, d_{4, i}\right) \mapsto\left(\underline{d}_{1}\right.$, $\left.\underline{d}_{2}, \underline{d}_{3}, \underline{d}_{4}\right) \in\left[D_{1}, \infty\right) \times\left[D_{2}, \infty\right) \times\left[D_{3}, \infty\right) \times\left[0, D_{4}\right]$. Let $i \mapsto \infty$ in (61); we obtain

$$
\begin{gathered}
\int_{\Omega}\left(r u_{1}-B u_{1}^{2}-m u_{1} u_{2}-p m u_{1} u_{3}\right) d x=0, \\
\int_{\Omega}\left(e m u_{1} u_{2}-a u_{2} u_{3}-\mu u_{2}\right) d x=0 \\
\int_{\Omega}\left(a u_{2} u_{3}+e m p u_{1} u_{3}-\delta u_{3}\right) d x=0 .
\end{gathered}
$$

Now, we consider the following three cases, respectively.

Case $1\left(u_{1} \equiv 0\right)$. Note that $u_{1 i} \mapsto u_{1}$, as $i \mapsto \infty$. Then we know that

$$
e m u_{1 i}-a u_{3 i}-\mu<0 \quad \text { on } \bar{\Omega}, \forall i \gg 1 .
$$

Integrating the differential equation for $u_{2 i}$ over $\Omega$ by parts, we have

$$
\begin{aligned}
0 & =-d_{2 i} \int_{\partial \Omega} \partial_{\nu} u_{2 i} d x=-d_{2 i} \int_{\Omega} \Delta u_{2 i} d x \\
& =\int_{\Omega} u_{2 i}\left(e m u_{1 i}-a u_{3 i}-\mu\right) d x<0, \quad \forall i \gg 1,
\end{aligned}
$$

which is a contradiction.

Case $2\left(u_{2} \equiv 0, u_{1} \neq 0\right.$ on $\left.\bar{\Omega}\right)$. By using Hopf boundary lemma, we know $u_{1}>0$ on $\bar{\Omega}$. Then $u_{1}$ and $u_{3}$ satisfy the following equation:

$$
\begin{gathered}
-\underline{d}_{1} \Delta u_{1}=u_{1}\left(r-B u_{1}-p m u_{3}\right) \quad \text { in } \Omega, \\
\partial_{v} u_{1}=0 \quad \text { on } \partial \Omega .
\end{gathered}
$$


Let $u_{1}\left(x_{0}\right)=\min _{\bar{\Omega}} u_{1}(x)$. It follows from Lemma 4 and (67) that

$$
r-B u_{1}\left(x_{0}\right)-p m u_{3}\left(x_{0}\right) \leq 0
$$

that is,

$$
u_{1}\left(x_{0}\right) \geq \frac{r}{B}-\frac{p m}{B} u_{3}\left(x_{0}\right) \geq \frac{r}{B}-\frac{p m \bar{M}_{3}}{B} .
$$

By using the assumption $(e m p / B)\left(r-p m \bar{M}_{3}\right)>\delta$, we know that

$$
e m p u_{1 i}-\delta>0 \quad \text { on } \bar{\Omega}, \forall i \gg 1 .
$$

Integrating the differential equation for $u_{3 i}$ over $\Omega$ by parts, we have

$$
\begin{aligned}
0 & =-d_{3 i} \int_{\partial \Omega}\left[\left(1+d_{4} u_{1 i}\right) \partial_{\nu} u_{3 i}+d_{4} u_{3 i} \partial_{\nu} u_{1 i}\right] d x \\
& =-d_{3 i} \int_{\Omega} \Delta\left(u_{3 i}+d_{4} u_{1 i} u_{3 i}\right) d x \\
& =\int_{\Omega} u_{3 i}\left(e m p u_{1 i}+a u_{2 i}-\delta\right) d x>0, \quad \forall i \gg 1
\end{aligned}
$$

which is a contradiction.

Case $3\left(u_{3} \equiv 0, u_{1} \neq 0\right.$, and $u_{2} \neq 0$ on $\left.\bar{\Omega}\right)$. By using Hopf boundary lemma, we know $u_{1}>0$ and $u_{2}>0$ on $\bar{\Omega}$.

Then $u_{1}$ and $u_{2}$ satisfy the following equation:

$$
\begin{gathered}
-\underline{d}_{1} \Delta u_{1}=u_{1}\left(r-B u_{1}-m u_{2}\right) \quad \text { in } \Omega, \\
\partial_{\nu} u_{1}=0 \quad \text { on } \partial \Omega .
\end{gathered}
$$

Let $u_{1}\left(x_{1}\right)=\min _{\bar{\Omega}} u_{1}(x)$. It follows from Lemma 4 and (72) that

$$
r-B u_{1}\left(x_{1}\right)-m u_{2}\left(x_{1}\right) \leq 0
$$

that is,

$$
u_{1}\left(x_{1}\right) \geq \frac{r}{B}-\frac{m}{B} u_{2}\left(x_{1}\right) \geq \frac{r}{B}-\frac{m \bar{M}_{2}}{B} .
$$

By using the assumption $(e m p / B)\left(r-m \bar{M}_{2}\right)>\delta$, we know that

$$
e m p u_{1 i}-\delta>0 \quad \text { on } \bar{\Omega}, \forall i \gg 1 .
$$

Integrating the differential equation for $u_{3 i}$ over $\Omega$ by parts, we have

$$
\begin{aligned}
0 & =-d_{3 i} \int_{\partial \Omega}\left[\left(1+d_{4} u_{1 i}\right) \partial_{\nu} u_{3 i}+d_{4} u_{3 i} \partial_{\nu} u_{1 i}\right] d x \\
& =-d_{3 i} \int_{\Omega} \Delta\left(u_{3 i}+d_{4} u_{1 i} u_{3 i}\right) d x \\
& =\int_{\Omega} u_{3 i}\left(e m p u_{1 i}+a u_{2 i}-\delta\right) d x>0, \quad \forall i \gg 1,
\end{aligned}
$$

which is a contradiction. The proof is completed.

\section{Nonexistence of Nonconstant Positive Solution of System (3) without Cross-Diffusion}

In order to discuss the effect of cross-diffusion on the existence of nonconstant positive solution of system (3), we first give a nonexistence result when the cross-diffusion term is absent, which shows that the cross-diffusion coefficients do play important roles. The mathematical technique to be employed here is the energy method.

Theorem 9. Suppose that $d_{4}=0$ and $d_{1} \geq r / \mu_{2} \triangleq D_{1}^{*}$, where $\mu_{2}$ is given in Notation 1. There exist positive constants $D_{2}^{*}$ and $D_{3}^{*}$, depending on $\Lambda, \varepsilon, \Omega$ such that (3) has no nonconstant positive solution provided that $d_{2} \geq D_{2}^{*}$ and $d_{3} \geq D_{3}^{*}$. Furthermore, one has

$$
u_{i} \equiv \bar{u}_{i}, \quad \text { where } \bar{u}_{i}=\frac{1}{\left|u_{i}\right|} \int_{\Omega} u_{i} d x, i=1,2,3 .
$$

Proof. Assume that $\mathbf{u}=\left(u_{1}, u_{2}, u_{3}\right)$ is a positive solution of (3) with $d_{4}=0$. Multiplying the $i$ th equation of (3) by $u_{i}-\bar{u}_{i}$ and integrating the results over $\Omega$ by parts, we have

$$
\begin{aligned}
& d_{1} \int_{\Omega}\left|\nabla u_{1}\right|^{2} d x=\int_{\Omega}\left(u_{1}-\bar{u}_{1}\right) \\
& \times\left(r u_{1}-B u_{1}^{2}-m u_{1} u_{2}-p m u_{1} u_{3}\right. \\
&-r \bar{u}_{1}+B \bar{u}_{1}^{2}+m \bar{u}_{1} \bar{u}_{2} \\
&\left.+p m \bar{u}_{1} \bar{u}_{3}\right) d x \\
&=\int_{\Omega}\left(u_{1}-\bar{u}_{1}\right)^{2} \\
& \quad \times\left(r-B u_{1}-B \bar{u}_{1}-m u_{2}-p m u_{3}\right) d x
\end{aligned}
$$

$$
\begin{aligned}
& -\int_{\Omega} m \bar{u}_{1}\left(u_{1}-\bar{u}_{1}\right)\left(u_{2}-\bar{u}_{2}\right) d x \\
& -\int_{\Omega} p m \bar{u}_{1}\left(u_{1}-\bar{u}_{1}\right)\left(u_{3}-\bar{u}_{3}\right) d x ;
\end{aligned}
$$

$$
\begin{aligned}
& d_{2} \int_{\Omega}\left|\nabla u_{2}\right|^{2} d x= \int_{\Omega}\left(u_{2}-\bar{u}_{2}\right) \\
& \times\left(e m u_{1} u_{2}-a u_{2} u_{3}-\mu u_{2}\right. \\
&\left.-e m \bar{u}_{1} \bar{u}_{2}+a \bar{u}_{2} \bar{u}_{3}+\mu \bar{u}_{2}\right) d x \\
&=\int_{\Omega}\left(u_{2}-\bar{u}_{2}\right)^{2}\left(e m u_{1}-a u_{3}-\mu\right) d x \\
&+\int_{\Omega} e m \bar{u}_{2}\left(u_{1}-\bar{u}_{1}\right)\left(u_{2}-\bar{u}_{2}\right) d x \\
&-\int_{\Omega} a \bar{u}_{2}\left(u_{2}-\bar{u}_{2}\right)\left(u_{3}-\bar{u}_{3}\right) d x
\end{aligned}
$$




$$
\begin{aligned}
& d_{3} \int_{\Omega}\left|\nabla u_{3}\right|^{2} d x= \int_{\Omega}\left(u_{3}-\bar{u}_{3}\right) \\
& \times\left(e m p u_{1} u_{3}+a u_{2} u_{3}-\delta u_{3}\right. \\
&\left.-e m p \bar{u}_{1} \bar{u}_{3}-a \bar{u}_{2} \bar{u}_{3}+\delta \bar{u}_{2}\right) d x \\
&=\int_{\Omega}\left(u_{3}-\bar{u}_{3}\right)^{2}\left(e m p u_{1}-a u_{2}-\delta\right) d x \\
&+\int_{\Omega} e m p \bar{u}_{3}\left(u_{1}-\bar{u}_{1}\right)\left(u_{3}-\bar{u}_{3}\right) d x \\
&+\int_{\Omega} a \bar{u}_{2}\left(u_{2}-\bar{u}_{2}\right)\left(u_{3}-\bar{u}_{3}\right) d x .
\end{aligned}
$$

Then it follows from (78) that

$$
\begin{aligned}
d_{1} \int_{\Omega}\left|\nabla u_{1}\right|^{2} d x+d_{2} \int_{\Omega}\left|\nabla u_{2}\right|^{2} d x+d_{3} \int_{\Omega}\left|\nabla u_{3}\right|^{2} d x \\
=\int_{\Omega}\left(u_{1}-\bar{u}_{1}\right)^{2} \\
\quad \times\left(r-B u_{1}-B \bar{u}_{1}-m u_{2}-p m u_{3}\right) d x \\
+\int_{\Omega}\left(u_{2}-\bar{u}_{2}\right)^{2}\left(e m u_{1}-a u_{3}-\mu\right) d x \\
+\int_{\Omega}\left(u_{3}-\bar{u}_{3}\right)^{2}\left(e m p u_{1}-a u_{2}-\delta\right) d x \\
+\int_{\Omega}\left(e m \bar{u}_{2}-m \bar{u}_{1}\right)\left(u_{1}-\bar{u}_{1}\right)\left(u_{2}-\bar{u}_{2}\right) d x \\
+\int_{\Omega}\left(e m p \bar{u}_{3}-p m \bar{u}_{1}\right)\left(u_{1}-\bar{u}_{1}\right)\left(u_{3}-\bar{u}_{3}\right) d x \\
+\int_{\Omega}\left(a \bar{u}_{3}-a \bar{u}_{2}\right)\left(u_{2}-\bar{u}_{2}\right)\left(u_{3}-\bar{u}_{3}\right) d x .
\end{aligned}
$$

By Cauchy inequality with $\varepsilon$, we can get from (79) that

$$
\begin{gathered}
d_{1} \int_{\Omega}\left|\nabla u_{1}\right|^{2} d x+d_{2} \int_{\Omega}\left|\nabla u_{2}\right|^{2} d x+d_{3} \int_{\Omega}\left|\nabla u_{3}\right|^{2} d x \\
\leq \int_{\Omega}\left(u_{1}-\bar{u}_{1}\right)^{2} \\
\quad \times\left(r-B u_{1}-B \bar{u}_{1}-m u_{2}-p m u_{3}+2 \varepsilon\right) d x \\
+\int_{\Omega}\left(u_{2}-\bar{u}_{2}\right)^{2} \\
\times\left(e m u_{1}-a u_{3}-\mu+\frac{\left(e m \bar{u}_{2}-m \bar{u}_{1}\right)^{2}}{4 \varepsilon}\right. \\
\left.+\frac{\left(a \bar{u}_{3}-a \bar{u}_{2}\right)^{2}}{4 \varepsilon}\right) d x
\end{gathered}
$$

$$
\begin{aligned}
& +\int_{\Omega}\left(u_{3}-\bar{u}_{3}\right)^{2} \\
& \times\left(e m p u_{1}-a u_{2}-\delta+\frac{\left(e m p \bar{u}_{3}-m p \bar{u}_{1}\right)^{2}}{4 \varepsilon}\right. \\
& \left.+\frac{\left(a \bar{u}_{3}-a \bar{u}_{2}\right)^{2}}{4 \varepsilon}\right) d x .
\end{aligned}
$$

On the other hand, applying Poincaré inequality, we know that

$$
\begin{gathered}
d_{1} \int_{\Omega}\left|\nabla u_{1}\right|^{2} d x+d_{2} \int_{\Omega}\left|\nabla u_{2}\right|^{2} d x+d_{3} \int_{\Omega}\left|\nabla u_{3}\right|^{2} d x \\
\geq \int_{\Omega} d_{1} \mu_{2}\left(u_{1}-\bar{u}_{1}\right)^{2}+\int_{\Omega} d_{2} \mu_{2}\left(u_{2}-\bar{u}_{2}\right)^{2} \\
+\int_{\Omega} d_{3} \mu_{2}\left(u_{3}-\bar{u}_{3}\right)^{2} .
\end{gathered}
$$

Then, by assumption, we can choose a sufficiently small positive constant $\varepsilon_{0}$ such that

$$
d_{1} \mu_{2}>r-B u_{1}-B \bar{u}_{1}-m u_{2}-p m u_{3}+2 \varepsilon_{0} .
$$

So by taking

$$
\begin{gathered}
D_{2}^{*}>\frac{1}{\mu_{2}}\left(e m u_{1}-a u_{3}-\mu\right. \\
\left.+\frac{\left(e m \bar{u}_{2}-m \bar{u}_{1}\right)^{2}}{4 \varepsilon_{0}}+\frac{\left(a \bar{u}_{3}-a \bar{u}_{2}\right)^{2}}{4 \varepsilon_{0}}\right), \\
D_{3}^{*}>\frac{1}{\mu_{2}}\left(e m p u_{1}-a u_{2}-\delta\right. \\
+\frac{\left(e m p \bar{u}_{3}-m p \bar{u}_{1}\right)^{2}}{4 \varepsilon_{0}} \\
\left.+\frac{\left(a \bar{u}_{3}-a \bar{u}_{2}\right)^{2}}{4 \varepsilon_{0}}\right)
\end{gathered}
$$

we can conclude that, when $d_{4}=0,(3)$ has only the positive constant solution $u_{i} \equiv \bar{u}_{i}$ for $i=1,2,3$. The proof is completed.

\section{Existence of Nonconstant Positive Solution of System (3)}

From Theorem 9 we know that, when the cross-diffusion $d_{3} d_{4} u_{1} u_{3}$ is absent, (3) has no nonconstant positive solution under some conditions. In the following, we will discuss the effect of cross-diffusion on the existence of nonconstant positive solution of system (3) for certain values of diffusion coefficient $d_{3}$, while the other parameters are fixed.

Our main findings are the following theorem, which shows that the presence of cross-diffusion creates nonhomogeneous solution. 
Theorem 10. Let $d_{1}, d_{2}$, and $d_{4}$ be fixed and satisfy (33) and (58), and let $\mu_{2}^{*}$ and $\mu_{3}^{*}$ be defined in (34). If $\mu_{2}^{*} \in\left(\mu_{i}, \mu_{i+1}\right)$ and $\mu_{3}^{*} \in\left(\mu_{j}, \mu_{j+1}\right)$ for some $j>i \geq 1$ and the sum $\sum_{n=i+1}^{j} m\left(\mu_{n}\right)$ is odd, then there exists a positive constant $d_{3}^{*}$ such that, if $d \geq d_{3}^{*}$, (3) admits at least one nonconstant positive solution.

In order to prove the above theorem by using LeraySchauder theory, we start with some preliminary results. Throughout this section, Notation 1 and $\mathbf{G}(\mathbf{u})$ defined in Section 2 will be used again. Define the set

$$
\begin{gathered}
\mathbf{X}^{+}=\left\{\mathbf{u}=\left(u_{1}, u_{1}, u_{1}\right)^{T} \in \mathbf{X}: u_{i}>0 \text { on } \bar{\Omega}, i=1,2,3\right\}, \\
\mathbf{B}(\mathbf{C})=\left\{\mathbf{u}=\left(u_{1}, u_{1}, u_{1}\right)^{T}, \underline{C} \leq u_{i} \leq \bar{C} \text { on } \bar{\Omega}, i=1,2,3\right\},
\end{gathered}
$$

where $\bar{C}=\max \left\{\bar{M}_{1}, \bar{M}_{2}, \bar{M}_{3}\right\}$ and $\underline{C}$ is given in Theorem 8 . Then we will look for nonconstant positive solutions of (3) in the set $\mathbf{B}(\mathbf{C})$. Let $\Phi(\mathbf{u})=\left[d_{1} u_{1}, d_{2} u_{2}, d_{3}\left(u_{3}+d_{4} u_{1} u_{3}\right)\right]^{T}$. Then (3) can be written as

$$
\begin{gathered}
-\Delta \Phi(\mathbf{u})=\mathbf{G}(\mathbf{u}) \quad \text { in } \Omega, \\
\partial_{\gamma} \mathbf{u}=0 \quad \text { on } \partial \Omega .
\end{gathered}
$$

Noting that the determinant of $\Phi_{\mathbf{u}}(\mathbf{u})$ is positive for all $\mathbf{u} \in$ $\mathbf{X}^{+}$, we know that $\Phi_{\mathbf{u}}^{-1}(\mathbf{u})$ exists and $\operatorname{det} \Phi_{\mathbf{u}}^{-1}(\mathbf{u})$ is positive. Then, $\mathbf{u}$ is a positive solution to (85) if and only if

$$
\begin{aligned}
& \mathbf{F}(\mathbf{u})= \mathbf{u}-(\mathbf{I}-\Delta)^{-1} \\
& \times\left\{\Phi_{\mathbf{u}}^{-1}(\mathbf{u})\left[\mathbf{G}(\mathbf{u})+\nabla \mathbf{u} \Phi_{u u}(\mathbf{u}) \nabla \mathbf{u}+\mathbf{u}\right]\right\}=0 \\
& \text { in } \mathbf{X}^{+},
\end{aligned}
$$

where $(\mathbf{I}-\Delta)^{-1}$ is the inverse of $\mathbf{I}-\Delta$ in $\mathbf{X}$ with the noflux boundary condition. As $\mathbf{F}(\cdot)$ is a compact perturbation of the identity operator, for any $\mathbf{B}=\mathbf{B}(\mathbf{C})$, the Leray-Schauder degree $\operatorname{deg}(\mathbf{F}(\cdot), 0, \mathbf{B})$ is well defined if $\mathbf{F}(\mathbf{u}) \neq 0$ on $\partial \mathbf{B}$. Note that

$$
\mathbf{D}_{\mathbf{u}} \mathbf{F}\left(\mathbf{u}^{*}\right)=\mathbf{I}-(\mathbf{I}-\Delta)^{-1}\left\{\Phi_{\mathbf{u}}^{-1}\left(\mathbf{u}^{*}\right)\left[\mathbf{G}_{\mathbf{u}}\left(\mathbf{u}^{*}\right)+\mathbf{I}\right]\right\}
$$

If $\mathbf{D}_{\mathbf{u}} \mathbf{F}\left(\mathbf{u}^{*}\right)$ is invertible, the index of $\mathbf{F}$ at $\mathbf{u}^{*}$ is defined as index $\left(\mathbf{F}(\cdot), \mathbf{u}^{*}\right)=(-1)^{\gamma}$, where $\gamma$ is the multiplicity of negative eigenvalues of $\mathbf{D}_{\mathbf{u}} \mathbf{F}\left(\mathbf{u}^{*}\right)$ [40, Theorem 2.8.1]. For the sake of convenience, we denote

$$
\begin{aligned}
\mathbf{H}(\mu) & =\mathbf{H}\left(\mathbf{u}^{*}, \mu\right) \triangleq \operatorname{det}\left[\mu \mathbf{I}-\Phi_{\mathbf{u}}^{-1}\left(\mathbf{u}^{*}\right) \mathbf{G}_{\mathbf{u}}\left(\mathbf{u}^{*}\right)\right] \\
& =\operatorname{det}\left[\Phi_{\mathbf{u}}^{-1}\left(\mathbf{u}^{*}\right)\right] \operatorname{det}\left[\mu \Phi_{\mathbf{u}}\left(\mathbf{u}^{*}\right)-\mathbf{G}_{\mathbf{u}}\left(\mathbf{u}^{*}\right)\right]
\end{aligned}
$$

By arguments similar to those in [41], we can conclude that the following proposition holds.

Proposition 11. Suppose that, for all $n \geq 1$, the matrix $\mu_{n} \mathbf{I}-$ $\Phi_{\mathbf{u}}^{-1}\left(\mathbf{u}^{*}\right) \mathbf{G}_{\mathbf{u}}\left(\mathbf{u}^{*}\right)$ is nonsingular. Then index $\left(\mathbf{F}(\cdot), \mathbf{u}^{*}\right)=(-1)^{\sigma}$, where $\sigma=\sum_{n \geq 1, \mathbf{H}\left(\mu_{n}\right)<0} \operatorname{dim} S\left(\mu_{n}\right)$.
From Proposition 11, we can see that, in order to compute index $\left(\mathbf{F}(\cdot), \mathbf{u}^{*}\right)$, it is necessary to consider carefully the sign of $\mathbf{H}\left(\mu_{i}\right)$. Noting that $\operatorname{det} \Phi_{\mathbf{u}}^{-1}\left(\mathbf{u}^{*}\right)$ is positive, then we only need to consider the sign of $\operatorname{det}\left[\mu \Phi_{\mathbf{u}^{*}}\left(\mathbf{u}^{*}\right)-\mathbf{G}_{\mathbf{u}}\left(\mathbf{u}^{*}\right)\right]$. In fact, the direct calculation gives that the value of $\bar{c}_{3}$, which is given in (30), is equal to $\operatorname{det}\left[\mu \Phi_{\mathbf{u}^{*}}\left(\mathbf{u}^{*}\right)-\mathbf{G}_{\mathbf{u}}\left(\mathbf{u}^{*}\right)\right]$. To study the existence of the positive solution of (3) with respect to the cross-diffusion constant $d_{3} d_{4}$, we will concentrate on the dependence of $\mathbf{H}\left(\mu_{i}\right)$ on $d_{3}$, and let $d_{1}, d_{2}$, and $d_{4}$ be fixed. Hence, from Theorem 3, we first introduce the following proposition.

Proposition 12. Assume that $d_{4}>0$ and that (33) holds. Then there exists a positive number $d_{3}^{*}$ such that, for all $d_{3} \geq d_{3}^{*}$, all the three roots $\tilde{\mu}_{1}, \tilde{\mu}_{2}$, and $\tilde{\mu}_{3}$ of $\operatorname{det}\left[\mu \Phi\left(\mathbf{u}^{*}\right)-\mathbf{G}_{\mathbf{u}}\left(\mathbf{u}^{*}\right)\right]=0$ are real and satisfy

$$
\begin{gathered}
-\infty<\widetilde{\mu}_{1}<0<\widetilde{\mu}_{2}<\widetilde{\mu}_{3} ; \\
\operatorname{det}\left[\mu \Phi_{\mathbf{u}}\left(\mathbf{u}^{*}\right)-\mathbf{G}_{\mathbf{u}}\left(\mathbf{u}^{*}\right)\right]<0, \\
\text { if } \mu \in\left(-\infty, \widetilde{\mu}_{1}\right) \cup\left(\widetilde{\mu}_{2}, \widetilde{\mu}_{3}\right) ; \\
\operatorname{det}\left[\mu \Phi_{\mathbf{u}}\left(\mathbf{u}^{*}\right)-\mathbf{G}_{\mathbf{u}}\left(\mathbf{u}^{*}\right)\right]>0, \\
\text { if } \mu \in\left(\widetilde{\mu}_{1}, \widetilde{\mu}_{2}\right) \cup\left(\widetilde{\mu}_{3}, \infty\right) .
\end{gathered}
$$

Proof of Theorem 10. By Proposition 12 and our assumptions, there exists a positive constant $d_{3}^{*}$ such that, when $d_{3} \geq d_{3}^{*}$, (89) holds and

$$
\mu_{i}<\tilde{\mu}_{2}<\mu_{i+1}, \quad \mu_{j}<\widetilde{\mu}_{3}<\mu_{j+1} .
$$

Now, we show that, for any $d_{3} \geq d_{3}^{*}$, (3) has at least one nonconstant positive solution. The proof, which will be accomplished by a contradict argument, is based on the homotopy invariance of the topological degree.

Suppose, on the contrary, that the assertion is not true. Let $\widehat{d}_{i}=D_{i}^{*}(i=1,2,3,4)$, where $D_{i}^{*}$ is defined in Theorem 9. For $t \in[0,1]$, define

$$
\begin{gathered}
\Phi(t ; \mathbf{u})=\left[\widehat{d}_{1} u_{1}+t\left(d_{1}-\widehat{d}_{1}\right) u_{1}, \widehat{d}_{2} u_{2}+t\left(d_{2}-\widehat{d}_{2}\right) u_{2},\right. \\
\left.\widehat{d}_{3}+t\left(d_{3}-\widehat{d}_{3}\right)\left(u_{3}+t d_{4} u_{1} u_{3}\right)\right]^{T} .
\end{gathered}
$$

Now we consider the following problem:

$$
\begin{gathered}
-\Delta \Phi(t ; \mathbf{u})=\mathbf{G}(\mathbf{u}) \quad \text { in } \Omega, 0 \leq t \leq 1, \\
\partial_{\nu} \mathbf{u}=0 \quad \text { on } \partial \Omega .
\end{gathered}
$$

Then $\mathbf{u}$ is a positive solution of ( 3 ) if and only if it is a positive solution of (92) for $t=1$. For $0 \leq t \leq 1$, it is obvious that $\mathbf{u}^{*}$ is the unique positive constant solution of (92) and $\mathbf{u}$ is a positive solution of (92) if and only if

$$
\begin{aligned}
\mathbf{F}(t ; \mathbf{u})= & \mathbf{u}-(\mathbf{I}-\Delta)^{-1} \\
& \times\left\{\Phi_{\mathbf{u}}^{-1}(t ; \mathbf{u})\left[\mathbf{G}(\mathbf{u})+\nabla \mathbf{u} \Phi_{u u}(t ; \mathbf{u}) \nabla \mathbf{u}\right]+\mathbf{u}\right\}=0 \\
& \text { in } \mathbf{X}^{+} .
\end{aligned}
$$


Clearly, $\mathbf{F}(1 ; \mathbf{u})=\mathbf{F}(\mathbf{u})$. Theorem 9 shows that the only positive solution of $\mathbf{F}(0 ; \mathbf{u})$ is $\mathbf{u}^{*}$ in $\mathbf{B}(\mathbf{C})$. By a direct computation, we have

$$
\mathbf{D}_{\mathbf{u}} \mathbf{F}\left(\mathbf{t} ; \mathbf{u}^{*}\right)=\mathbf{I}-(\mathbf{I}-\Delta)^{-1}\left\{\Phi_{\mathbf{u}}^{-1}\left(t ; \mathbf{u}^{*}\right) \mathbf{G}_{\mathbf{u}}\left(\mathbf{u}^{*}\right)+\mathbf{I}\right\} \text {. }
$$

In particular,

$$
\begin{gathered}
\mathbf{D}_{\mathbf{u}} \mathbf{F}\left(\mathbf{0} ; \mathbf{u}^{*}\right)=\mathbf{I}-(\mathbf{I}-\Delta)^{-1}\left\{\mathfrak{D}^{-1} \mathbf{G}_{\mathbf{u}}\left(\mathbf{u}^{*}\right)+\mathbf{I}\right\}, \\
\mathbf{D}_{\mathbf{u}} \mathbf{F}\left(\mathbf{1} ; \mathbf{u}^{*}\right)=\mathbf{I}-(\mathbf{I}-\Delta)^{-1}\left\{\Phi_{\mathbf{u}}^{-1}\left(\mathbf{u}^{*}\right) \mathbf{G}_{\mathbf{u}}\left(\mathbf{u}^{*}\right)+\mathbf{I}\right\},
\end{gathered}
$$

where $\mathfrak{D}=\operatorname{diag}\left(\widehat{d}_{1}, \widehat{d}_{2}, \widehat{d}_{3}\right)$. In view of Proposition 12 and (90), it follows that

$$
\begin{gathered}
\mathbf{H}\left(\mu_{1}\right)=\mathbf{H}(0)>0, \\
\mathbf{H}\left(\mu_{n}\right)<0, \quad i+1 \leq n \leq j, \\
\mathbf{H}\left(\mu_{n}\right)>0, \quad 1<n \leq i \text { or } n \geq j+1 .
\end{gathered}
$$

Therefore, zero is not an eigenvalue of the matrix $\mu_{n} I-$ $\Phi_{\mathbf{u}}^{-1}\left(\mathbf{u}^{*}\right) \mathbf{G}_{\mathbf{u}}\left(\mathbf{u}^{*}\right)$ for all $n \geq 1$, and

$$
\sum_{n \geq 1, H\left(\mu_{n}\right)<0} m\left(\mu_{n}\right)=\sum_{n=i+1}^{j} m\left(\mu_{n}\right)=\text { an odd number. }
$$

Then Proposition 11 yields

$$
\text { index }\left(\mathbf{I}-\mathbf{F}(\mathbf{1} ; \cdot), \mathbf{u}^{*}\right)=(-1)^{\gamma}=-1 .
$$

Similarly we can get that

$$
\text { index }\left(\mathbf{I}-\mathbf{F}(\mathbf{0} ; \cdot), \mathbf{u}^{*}\right)=(-1)^{0}=1 \text {. }
$$

According to Theorems 9 and (40) and (59), there exist positive constants $\bar{C}$ and $\underline{C}$ such that, for all $0 \leq t \leq 1$, the positive solutions of (3) satisfy $\underline{C} \leq u_{1}, u_{2}, u_{3} \leq \bar{C}$. Therefore, $\mathbf{F}(t ; \mathbf{u}) \neq 0$ on $\partial \mathbf{B}(\mathbf{C})$ for all $0 \leq t \leq 1$. By the homotopy invariance of the topological degree, we have

$$
\operatorname{deg}(\mathbf{I}-\mathbf{F}(\mathbf{1} ; \cdot), \mathbf{0}, \mathbf{B}(\mathbf{C}))=\operatorname{deg}(\mathbf{I}-\mathbf{F}(\mathbf{0} ; \cdot), 0, \mathbf{B}(\mathbf{C})) .
$$

On the other hand, under our assumptions, the only positive solution of both $\mathbf{F}(\mathbf{1} ; \mathbf{u})=0$ and $\mathbf{F}(\mathbf{0} ; \mathbf{u})=0$ in $\mathbf{B}(\mathbf{C})$ is $\mathbf{u}^{*}$, and hence, by (98) and (99),

$$
\begin{aligned}
& \operatorname{deg}(\mathbf{I}-\mathbf{F}(\mathbf{0} ; \cdot), 0, \mathbf{B}(\mathbf{C}))=\operatorname{index}\left(\mathbf{I}-\mathbf{F}(\mathbf{0} ; \cdot), \mathbf{u}^{*}\right)=1, \\
& \operatorname{deg}(\mathbf{I}-\mathbf{F}(\mathbf{1} ; \cdot), 0, \mathbf{B}(\mathbf{C}))=\operatorname{index}\left(\mathbf{I}-\mathbf{F}(\mathbf{1} ; \cdot), \mathbf{u}^{*}\right)=-1,
\end{aligned}
$$

which contradicts (100). The proof is completed.

\section{Conflict of Interests}

The authors declare that there is no conflict of interests regarding the publication of this paper.

\section{Acknowledgments}

This work is supported by the National Science Foundation of China (11171276) and the Ph.D. Foundation of Southwest University (SWU112099).

\section{References}

[1] A. Casal, J. C. Eilbeck, and J. López-Gómez, "Existence and uniqueness of coexistence states for a predator-prey model with diffusion," Differential and Integral Equations, vol. 7, no. 2, pp. 411-439, 1994.

[2] R. Peng and J. P. Shi, "Non-existence of non-constant positive steady states of two Holling type-II predator-prey systems: strong interaction case," Journal of Differential Equations, vol. 247, no. 3, pp. 866-886, 2009.

[3] P. Y. H. Pang and M. X. Wang, "Non-constant positive steady states of a predator-prey system with non-monotonic functional response and diffusion," Proceedings of the London Mathematical Society, vol. 88, no. 1, pp. 135-157, 2004.

[4] Y. H. Du and Y. Lou, "Qualitative behaviour of positive solutions of a predator-prey model: effects of saturation," Proceedings of the Royal Society of Edinburgh A. Mathematics, vol. 131, no. 2, pp. 321-349, 2001.

[5] Y. H. Du and Y. Lou, "Some uniqueness and exact multiplicity results for a predator-prey model," Transactions of the American Mathematical Society, vol. 349, no. 6, pp. 2443-2475, 1997.

[6] M. X. Wang, "Stationary patterns for a prey-predator model with prey-dependent and ratio-dependent functional responses and diffusion," Physica D, vol. 196, no. 1-2, pp. 172-192, 2004.

[7] M. X. Wang, "Stationary patterns of strongly coupled preypredator models," Journal of Mathematical Analysis and Applications, vol. 292, no. 2, pp. 484-505, 2004.

[8] W. Ko and K. Ryu, "Coexistence states of a predator-prey system with non-monotonic functional response," Nonlinear Analysis. Real World Applications, vol. 8, no. 3, pp. 769-786, 2007.

[9] L. Li, "Coexistence theorems of steady states for predator-prey interacting systems," Transactions of the American Mathematical Society, vol. 305, no. 1, pp. 143-166, 1988.

[10] E. N. Dancer, "On positive solutions of some pairs of differential equations," Transactions of the American Mathematical Society, vol. 284, no. 2, pp. 729-743, 1984.

[11] J. Zhou and C. L. Mu, "Coexistence states of a Holling typeII predator-prey system," Journal of Mathematical Analysis and Applications, vol. 369, no. 2, pp. 555-563, 2010.

[12] M. Haque, "Ratio-dependent predator-prey models of interacting populations," Bulletin of Mathematical Biology, vol. 71, no. 2, pp. 430-452, 2009.

[13] M. Haque, "A predator-prey model with disease in the predator species only," Nonlinear Analysis. Real World Applications, vol. 11, no. 4, pp. 2224-2236, 2010.

[14] M. Haque and E. Venturino, "The role of transmissible diseases in Holling-Tanner predatorprey model," Theoretical Population Biology, vol. 70, pp. 273-288, 2006.

[15] M. Haque, J. Zhen, and E. Venturino, "An ecoepidemiological predator-prey model with standard disease incidence," Mathematical Methods in the Applied Sciences, vol. 32, no. 7, pp. 875898, 2009.

[16] Y. Xiao and L. Chen, "Analysis of a three species ecoepidemiological model," Journal of Mathematical Analysis and Applications, vol. 258, no. 2, pp. 733-754, 2001. 
[17] Y. Xiao and L. Chen, "Modeling and analysis of a predator-prey model with disease in the prey," Mathematical Biosciences, vol. 171, no. 1, pp. 59-82, 2001.

[18] H. W. Hethcote, W. Wang, and Z. Ma, "A predator-prey model with infected prey," Journal of Theoretical Population Biology, vol. 66, pp. 259-268, 2004.

[19] H. Malchow, S. V. Petrovskii, and E. Venturino, Spatiotemporal Patterns in Ecology and Epidemiology, CRC, 2008.

[20] E. Venturino, "The effects of diseases on competing species," Mathematical Biosciences, vol. 174, no. 2, pp. 111-131, 2001.

[21] E. Venturino, "The influence of diseases on Lotka-Volterra systems," The Rocky Mountain Journal of Mathematics, vol. 24, no. 1, pp. 381-402, 1994.

[22] E. Venturino, "Epidemics in predator-prey models: disease in the predators," IMA Journal of Mathematics Applied in Medicine and Biology, vol. 19, pp. 185-205, 2002.

[23] J. Chattopadhyay and O. Arino, "A predator-prey model with disease in the prey," Nonlinear Analysis. Theory, Methods \& Applications, vol. 36, no. 6, pp. 747-766, 1999.

[24] N. Shigesada, K. Kawasaki, and E. Teramoto, "Spatial segregation of interacting species," Journal of Theoretical Biology, vol. 79, no. 1, pp. 83-99, 1979.

[25] C. Tian, Z. Ling, and Z. Lin, "Turing pattern formation in a predator-prey-mutualist system," Nonlinear Analysis. Real World Applications, vol. 12, no. 6, pp. 3224-3237, 2011.

[26] Z. Xie, "Turing instability in a coupled predator-prey model with different Holling type functional responses," Discrete and Continuous Dynamical Systems S, vol. 4, no. 6, pp. 1621-1628, 2011.

[27] Z. Xie, "Cross-diffusion induced Turing instability for a three species food chain model," Journal of Mathematical Analysis and Applications, vol. 388, no. 1, pp. 539-547, 2012.

[28] R. Peng, M. Wang, and G. Yang, "Stationary patterns of the Holling-Tanner prey-predator model with diffusion and crossdiffusion," Applied Mathematics and Computation, vol. 196, no. 2, pp. 570-577, 2008.

[29] M. Haque, S. Sarwardi, S. Preston, and E. Venturino, "Effect of delay in a Lotka-Volterra type predator-prey model with a transmissible disease in the predator species," Mathematical Biosciences, vol. 234, no. 1, pp. 47-57, 2011.

[30] P. Y. H. Pang and M. Wang, "Strategy and stationary pattern in a three-species predator-prey model," Journal of Differential Equations, vol. 200, no. 2, pp. 245-273, 2004.

[31] M. X. Wang, "Stationary patterns for a prey-predator model with prey-dependent and ratio-dependent functional responses and diffusion," Physica D, vol. 196, no. 1-2, pp. 172-192, 2004.

[32] M. X. Wang, "Stationary patterns caused by cross-diffusion for a three-species prey-predator model," Computers \& Mathematics with Applications, vol. 52, no. 5, pp. 707-720, 2006.

[33] W. Chen and R. Peng, "Stationary patterns created by crossdiffusion for the competitor-competitor-mutualist model," Journal of Mathematical Analysis and Applications, vol. 291, no. 2, pp. 550-564, 2004.

[34] J.-F. Zhang, W.-T. Li, and Y.-X. Wang, "Turing patterns of a strongly coupled predator-prey system with diffusion effects," Nonlinear Analysis. Theory, Methods \& Applications, vol. 74, no. 3, pp. 847-858, 2011.

[35] Y. H. Xia and M. Han, "New conditions on the existence and stability of periodic solution in Lotka-Volterra's population system," SIAM Journal on Applied Mathematics, vol. 69, no. 6, pp. 1580-1597, 2009.
[36] Y. H. Xia, J. Cao, and M. Han, "A new analytical method for the linearization of dynamic equation on measure chains," Journal of Differential Equations, vol. 235, no. 2, pp. 527-543, 2007.

[37] D. Henry, Geometric Theory of Semilinear Parabolic Equations, vol. 840 of Lecture Notes in Mathematics, Springer, Berlin, Germany, 1981.

[38] Y. Lou and W.-M. Ni, "Diffusion, self-diffusion and crossdiffusion," Journal of Differential Equations, vol. 131, no. 1, pp. 79-131, 1996.

[39] C.-S. Lin, W.-M. Ni, and I. Takagi, "Large amplitude stationary solutions to a chemotaxis system," Journal of Differential Equations, vol. 72, no. 1, pp. 1-27, 1988.

[40] L. Nirenberg, Topics in Nonlinear Functional Analysis, vol. 6, American Mathematical Society, Providence, RI, USA, 2001.

[41] P. Y. H. Pang and M. X. Wang, "Qualitative analysis of a ratiodependent predator-prey system with diffusion," Proceedings of the Royal Society of Edinburgh A. Mathematics, vol. 133, no. 4, pp. 919-942, 2003. 


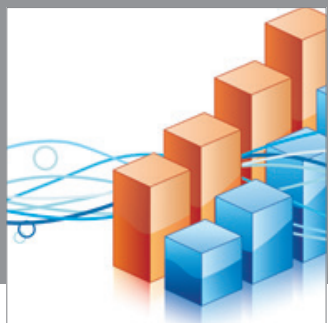

Advances in

Operations Research

mansans

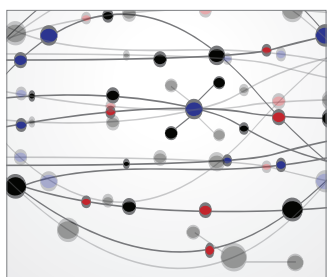

The Scientific World Journal
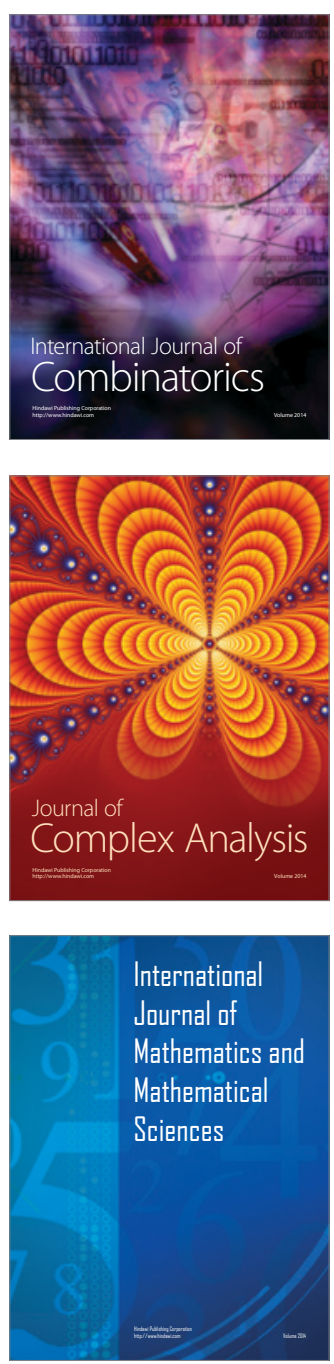
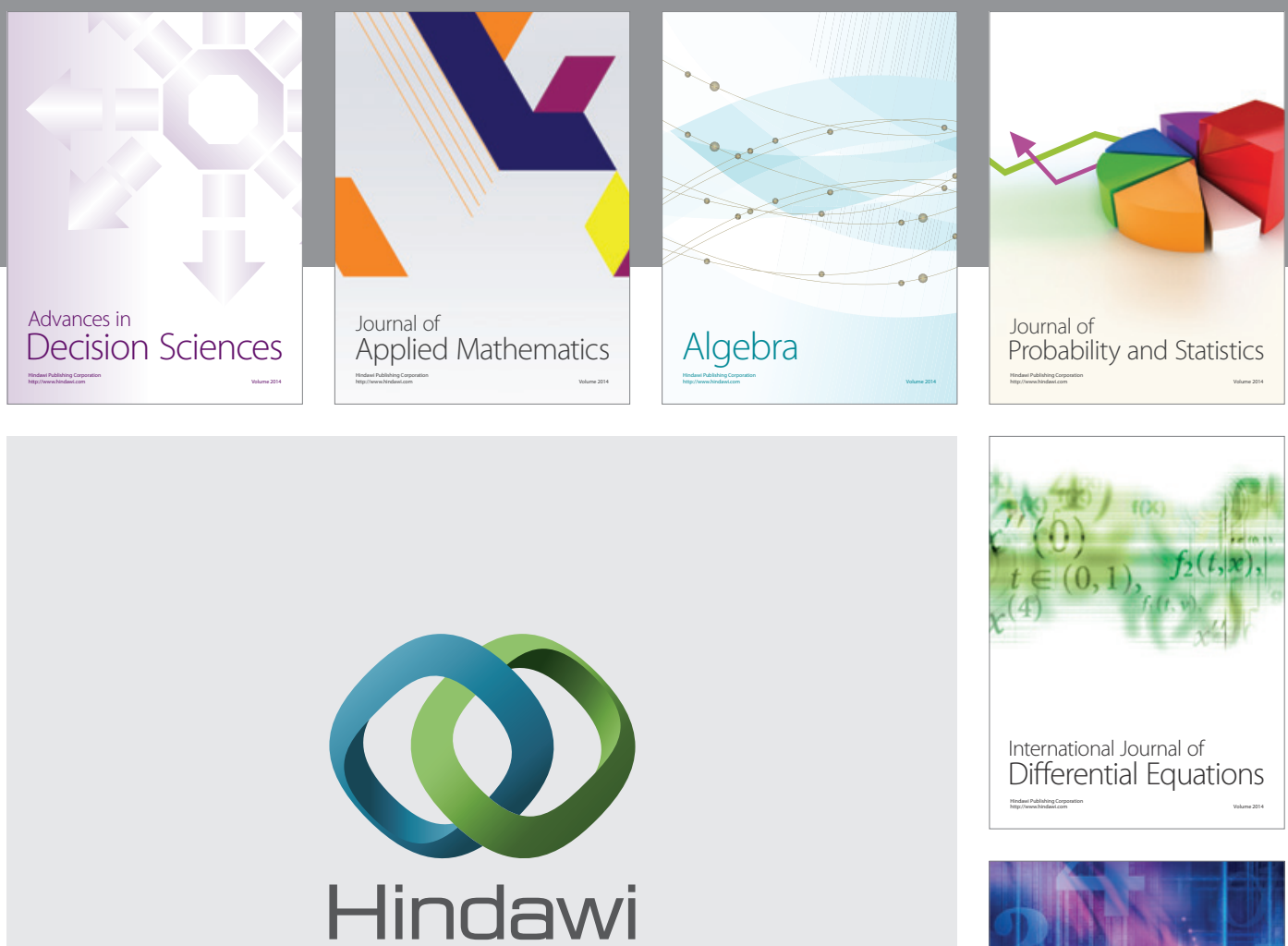

Submit your manuscripts at http://www.hindawi.com
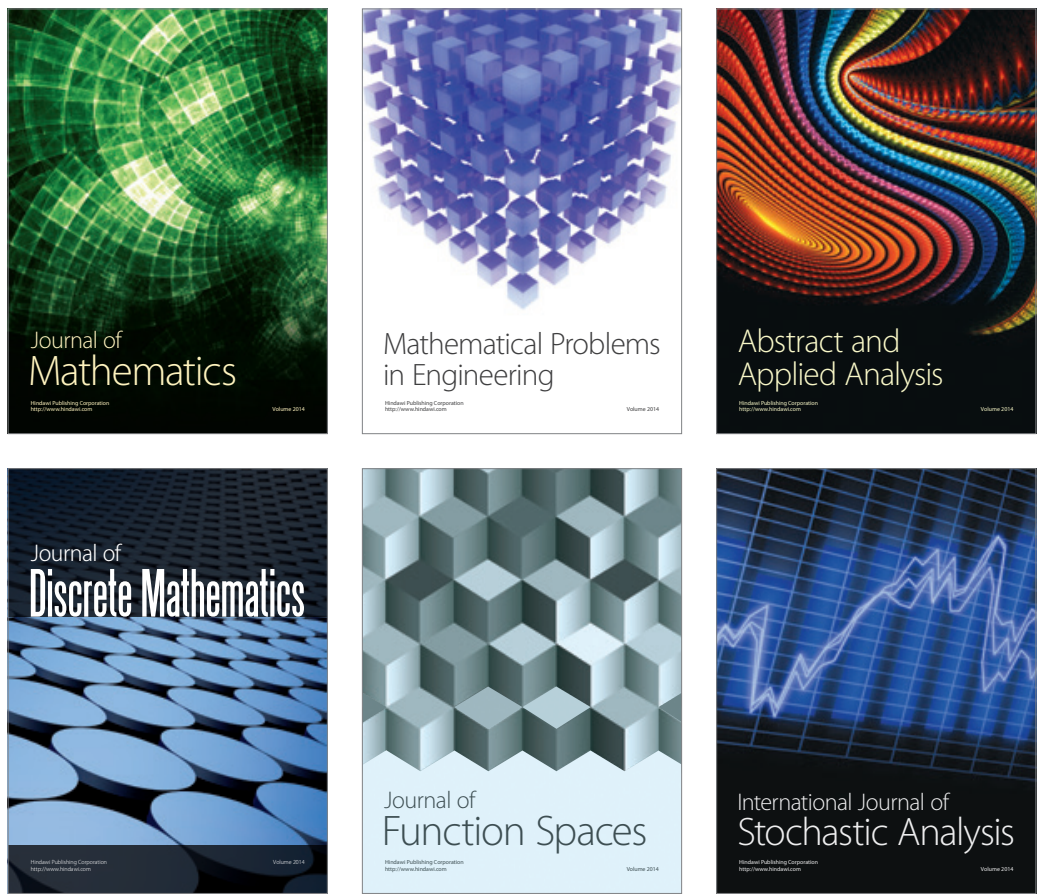

Journal of

Function Spaces

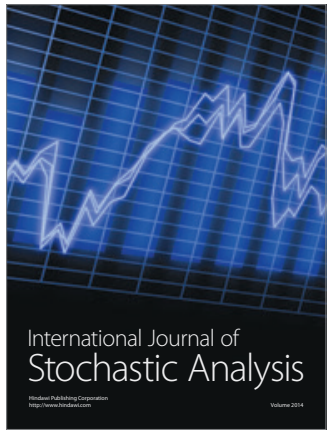

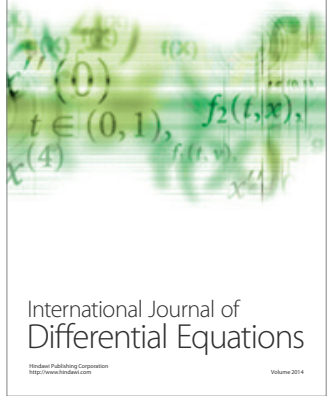
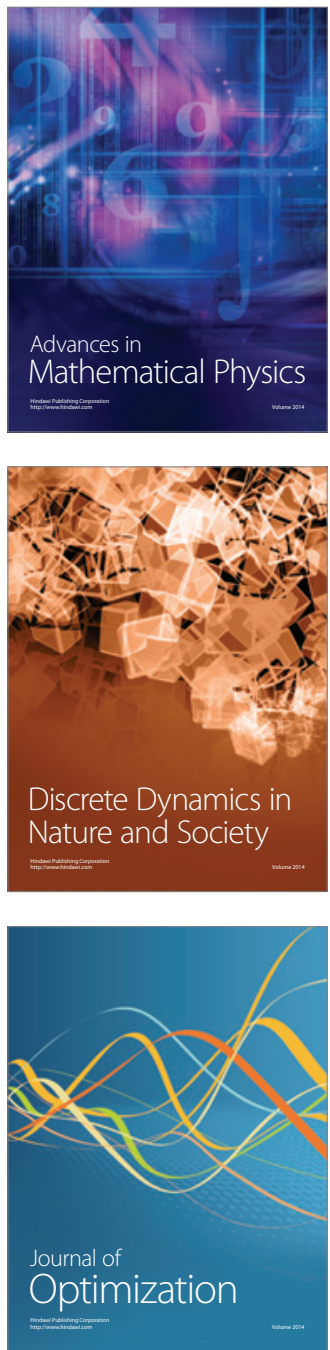